\title{
Review of: \\ Gene expression profiling identifies molecular subtypes of inflammatory breast cancer
}

\author{
P. E. Lønning \\ Section of Oncology, Department of Medicine, Haukeland University Hospital, Bergen, Norway.
}

\section{Citation of original article:}

F. Bertucci, P. Finetti, J. Rougemont, E. Charafe-Jauffret, N. Cervera, C. Tarpin, et al. Gene expression profiling identifies molecular subtypes of inflammatory breast cancer. Cancer Research 2005; 65(6): 2170-8.

\begin{abstract}
of the original article
Breast cancer is a heterogeneous disease. Comprehensive gene expression profiles obtained using DNA microarrays have revealed previously indistinguishable subtypes of non-inflammatory breast cancer (NIBC) related to different features of mammary epithelial biology and significantly associated with survival. Inflammatory breast cancer (IBC) is a rare, particular, and aggressive form of disease. Here we have investigated whether the five molecular subtypes described for NIBC (luminal A and B, basal, ERBB2 overexpressing, and normal breast-like) were also present in IBC. We monitored the RNA expression of approximately 8,000 genes in 83 breast tissue samples including 37 IBC, 44 NIBC, and 2 normal breast samples. Hierarchical clustering identified the five subtypes of breast cancer in both NIBC and IBC samples. These subtypes were highly similar to those defined in previous studies and associated with similar histoclinical features. The robustness of this classification was confirmed by the use of both alternative gene set and analysis method, and the results were corroborated at the protein level. Furthermore, we show that the differences in gene expression between NIBC and IBC and between IBC with and without pathologic complete response that we have recently reported persist in each subtype. Our results show that the expression signatures defining molecular subtypes of NIBC are also present in IBC. Obtained using different patient series and different microarray platforms, they reinforce confidence in the expression-based molecular taxonomy but also give evidence for its universality in breast cancer, independently of a specific clinical form.
\end{abstract}

\section{Review}

Inflammatory breast cancer (IBC) is the most aggressive variant of the disease with a distinct clinical presentation, microscopically characterized by tumour

Correspondence to: Professor Per E Lønning, Section on Oncology, Department of Medicine, Haukeland University Hospital, N-5021, Bergen, Norway

Received 05/07/05

Accepted 12/07/05

First published online 03/01/06

BCO/473/2005/JC emboli in lymphatic vessels, and a poor prognosis $[1,2]$. Similar to the group of Bieche et al. [3], Bertucci et al. last year published an interesting paper [4] revealing differences in gene expression profile between IBC and non-inflammatory breast cancer (NIBC) based on a list of 109 genes. They also provided a second list of 85 genes associated with pathological complete response in IBC. Here, in the same cohort of patients, they explored the subtype classification previously reported by Perou et al. and 
Sørlie et al. [5,6] for (NIBC) in inflammatory cancer. While this classification has been confirmed by others in NIBC [7], so far it has not been evaluated in IBC.

First, using the 500 gene list of Sørlie et al., they extracted 120 genes common to their gene list and the one used by Sørlie et al. They validated this list on the Sørlie dataset [6], predicting correct classification of $89 \%$ of the samples before analysing their own set of tumours (37 IBC and 44 NIBC). Analysing their 44 NIBC tumours, 32 of these samples could be classified into one of the five subclasses defined according to Sørlie et al. [6], while 12 tumours could not be classified. Surprisingly, among their 37 IBC, 36 could be classified according to this system. Most interestingly, while the total number does not allow statistical comparison, their finding of 14 and 3 tumours, respectively, in the luminal $A$ and $B$ class was unexpected, suggesting the incidence of tumours belonging to each of these classes is not much different in IBC compared with what has been recorded previously for NIBC. The surprise rest primarily on the knowledge that luminal A tumours in particular are associated with high expression of ER alpha, while IBC in general is known to express little responsiveness to hormonal manipulation.

Finally, Bertucci et al. compared the tumour classification according to Sørlie et al. to their own previously identified 109 gene list discriminating between IBC and NIBC as well as their 85 gene list, predicting responsiveness to chemotherapy in IBC. Importantly, they found that their previous gene lists were able to discriminate between IBC vs. NIBC as well as responsiveness to therapy across all tumour classes.

The findings by Bertucci et al. suggest some interesting biological interpretations. The 'molecular portraits' discriminating the different classes identified by Perou et al. and Sørlie et al. are likely due to early events in tumour development; the finding of a particular cytokeratin profile associated with the 'basal' subgroup may indicate a different cell of origin compared to the luminal tumours. Interestingly, in a recent paper Zhao et al. [8] were able to shown that among lobular carcinomas, about $50 \%$ of the tumours harboured a distinct gene profile different from all the subgroups identified by Perou et al. and Sørlie et al. for ductal carcinomas, while the other $50 \%$ could be separated into the subclasses identified for ductal cancers. Bieche et al. [3] found the major discriminators between IBC and NIBC to be genes associated with transcription, growth factors and growth factor receptors; the discriminators were uniformly up-regulated in IBC. Whether this could mean that achievement of an IBC profile could be a late event, related to mutations in genes critical to growth arrest that may occur, to some degree, independent of earlier events, is too early to say, but is definitely a possibility.
Similar to Sørlie et al. and Bertucci et al. found the tumour subclasses to be associated with prognosis, although the difference between the luminal A class and the other classes were not as distinct as in our material [6]. What needs to be emphasized however is that the tumours analysed in this study were all from patients treated in prospective protocols, incorporating administration of tamoxifen for 5 years to all patients harbouring a receptor positive tumour $[9,10]$. This may likely have improved outcome among patients with luminal $A$ tumours but not those with tumours belonging to the receptor negative classes, substantiated by the finding that the prognostic impact of the luminal A class was of a smaller magnitude among the node negative patients reported by the Amsterdam group that were not exposed to adjuvant therapy $[11,12]$. Use of adjuvant endocrine therapy was not accounted for in detail in the papers by Bertucci et al. $[4,13]$, and we lack information on whether the 'luminal A' gene profile is associated with hormone sensitivity in IBC.

The achievements through microarrays and gene profiling have up to now been encouraging but also disappointing. The list of conventional prognostic factors in breast cancer is long; what we currently are observing is an increasing list of molecular signatures identified by supervised clustering with limited overlap of genes $[14,15]$, the finding that multiple signatures may be derived from a single dataset [16] and the challenging question whether use of conventional factors in a combined index may provide prognostic information of similar value [17]. Although statistical associations between gene expression profiles and treatment outcome have been reported [4,18-21], they lack the sensitivity to be of clinical use selecting patients for therapy. To improve therapy, we need to explore not only statistical associations but to identify the biological mechanisms behind phenomenon as the metastatic process and drug resistance $[22,23]$. As such, this paper by Bertucci et al., together with the papers by Perou et al. and Sørlie et al., defining breast cancer subclasses, and the recent study by Glinsky et al. [24] reporting a stem-cell signature across tumour forms, may add information to our understanding of the biological mechanisms controlling vital processes in cancer development and behaviour.

\section{References}

1. Low JA, Berman AW, Steinberg SM, et al. Long-term follow-up for locally advanced and inflammatory breast cancer patients treated with multimodality therapy. J Clin Oncol 2004; 22: 4067-4074.

2. Jaiyesimi IA, Buzdar AU, Hortobagyi G. Inflammatory breast cancer: a review. J Clin Oncol 1992; 10: 1014-1024. 
3. Bieche I, Lerebours F, Tozlu S, et al. Molecular profiling of inflammatory breast cancer: identification of a poorprognosis gene expression signature. Clin Cancer Res 2004; 10: 6789-6795.

4. Bertucci F, Finetti P, Rougemont J, et al. Gene expression profiling for molecular characterization of inflammatory breast cancer and prediction of response to chemotherapy. Cancer Res 2004; 64: 8558-8565.

5. Perou CM, Sørlie T, Eisen MB, et al. Molecular portraits of human breast tumours. Nature 2000; 406: 747-752.

6. Sørlie T, Perou CM, Tibshirani R, et al. Gene expression patterns of breast carcinomas distinguish tumor subclasses with clinical implications. Proc Natl Acad Sci USA 2001; 98: 10869-10874.

7. Sotiriou C, Neo SY, McShane LM, et al. Breast cancer classification and prognosis based on gene expression profiles from a population-based study. Proc Natl Acad Sci USA 2003; 100: 10393-10398.

8. Zhao HJ, Langerod A, Ji Y, et al. Different gene expression patterns in invasive lobular and ductal carcinomas of the breast. Mol Biol Cell 2004; 15: 2523-2536.

9. Geisler S, Lønning PE, Aas T, et al. Influence of TP53 gene alterations and c-erbB-2 expression on the response to treatment with doxorubicin in locally advanced breast cancer. Cancer Res 2001; 61: 2505-2512.

10. Geisler S, Børresen-Dale A-L, Johnsen H, et al. TP53 gene mutations predict the response to neoadjuvant treatment with 5 fluorouracil and mitomycin in locally advanced breast cancer. Clin Cancer Res 2003; 9: 5582-5588.

11. van't Veer L, Dai H, van de Vijver M, et al. Gene expression profiling predicts clinical outcome of breast cancer. Nature 2002; 415: 530-536.

12. Sørlie T, Tibshirani R, Parker J, et al. Repeated observation of breast tumor subtypes in independent gene expression data sets. Proc Natl Acad Sci USA 2003; 100: 8418-8423.

13. Bertucci F, Finetti P, Rougemont J, et al. Gene expression profiling identifies molecular subtypes of inflammatory breast cancer. Cancer Res 2005; 65: 2170-2178.
14. van de Vijver MJ, He YD, van't Veer LJ, et al. A geneexpression signature as a predictor of survival in breast cancer. New Engl J Med 2002; 347: 1999-2009.

15. Wang Y, Klijn JG, Zhang Y, et al. Gene-expression profiles to predict distant metastasis of lymph-node-negative primary breast cancer. Lancet 2005; 365: 671-679.

16. Ein-Dor L, Kela I, Getz G, Givol D, Domany E. Outcome signature genes in breast cancer: is there a unique set? Bioinformatics 2005; 21: 171-178.

17. Edén P, Ritz C, Rose C, Fernö M, Peterson C. 'Good Old' clinical markers have similar power in breast cancer prognosis as microarray gene expression profilers. Eur $J$ Cancer 2004; 40: 1837-1841.

18. Chang J, Wooten E, Tsimelzon A, et al. Gene expression profiling for the prediction of therapeutic response to docetaxel in patients with breast cancer. Lancet 2003; 362: 362-369.

19. Ayers M, Symmans WF, Stec J, et al. Gene expression profiles predict complete pathologic response to neoadjuvant paclitaxel and fluorouracil, doxorubicin, and cyclophosphamide chemotherapy in breast cancer. J Clin Oncol 2004; 22: 2284-2293.

20. Hannemann J, Oosterkamp HM, Bosch CAJ, et al. Changes in gene expression associated with response to neoadjuvant chemotherapy in breast cancer. J Clin Oncol 2005; 23: 3331-3342.

21. Iwao-Koizumi K, Matoba R, Ueno N, et al. Prediction of docetaxel response in human breast cancer by gene expression profiling. J Clin Oncol 2005; 23: 422-431.

22. Lønning PE. Genes causing inherited cancer as beacons identifying the mechanisms of chemoresistance. Trends Mol Med 2004; 10: 113-118.

23. Lønning PE, Sørlie T, Børresen-Dale A-L. Genomics in breast cancer - therapeutic implications? Nature Clin Pract Oncol 2005; 2: 26-33.

24. Glinsky GV, Berezovska O, Glinskii AB. Microarray analysis identifies a death-from-cancer signature predicting therapy failure in patients with multiple types of cancer. $J$ Clin Invest 2005; 115: 1503-1521. 